\title{
Ascariasis hepatobiliar en una lactante con desnutrición severa: reporte de caso
}

\author{
Angie Bello-Suárez* \\ Gina González-Valencia* \\ Luis Sosa Ávila** \\ Patrik Sarmiento-Wilches***
}

\begin{abstract}
* Médica Pediatra. Universidad Industrial de Santander. Bucaramanga. Santander. Colombia.
**Médico Pediatra. Infectólogo. Departamento de Pediatría. Universidad Industrial de Santander. Hospital Universitario de Santander. Bucaramanga. Santander . Colombia.

***Médica Pediatra. Infectóloga. Clínica Materno Infantil San Luis. Bucaramanga. Santander. Colombia.

Correspondencia: Dra. Patrik Eliana Sarmiento Wilches. Dirección: Cra. 30 \#34-54 Hospital Universitario de Santander, piso 10, oficina del Departamento de Pediatría. Bucaramanga. Colombia. Teléfono: +57-6344000 Ext. 3102 - 3302. Correo electrónico: pesw5@hotmail.com.
\end{abstract}

\section{Resumen}

La Ascariasis es la geohelminitiasis más común del mundo, catalogándose como una enfermedad tropical desatendida, que puede causar compromiso pulmonar, gastrointestinal, hepatobiliar y nutricional. Se presenta el caso inusual de una lactante procedente de una zona de extrema pobreza quien consultó por fiebre, vómito, ausencia de deposiciones, distensión y dolor abdominal. Fue diagnosticada con pseudoobstrucción intestinal, desnutrición severa, choque séptico de origen intraabdominal, retraso del neurodesarrollo y deprivación psicoafectiva, cuyas imágenes reportaron ascariasis hepatobiliar y granulomas hepáticos calcificados y abscedados, con infestación severa por Ascaris lumbricoides. Recibió manejo antibiótico y antiparasitario con recuperación exitosa.

En nuestro medio, las infecciones por helmintos son causa frecuente de anemia, retraso cognitivo y del crecimiento en niños en edad escolar. Sin embargo, la infestación y complicaciones hepatobiliares como colangitis, colecistitis, pancreatitis, litiasis biliar y hepatitis abscedada son inusuales en menores de dos años. A través de este caso se pretende resaltar la presentación atípica de la enfermedad en lactantes e incitar al fortalecimiento de las intervenciones en salud pública. MÉD.UIS.2020;33(1):67-72.

Palabras claves: Ascaris lumbricoides. Enfermedades de las vías biliares. Desnutrición.

\section{Hepatobiliary ascariasis in severely malnourished infant: case report}

Abstract

Ascariasis is the most common geohelminitiasis in the world. It is categorized as an unattended tropical disease, which can cause pulmonary, gastrointestinal, hepatobiliary and nutritional compromise. We present the unusual case of an infant from an extreme poverty area presenting fever, vomiting, absence of bowel movements, bloating and abdominal pain. She was diagnosed with intestinal pseudoobstruction, severe malnutrition, abdominal septic shock, neurodevelopmental delay and emotional deprivation. The images reported hepatobiliary ascariasis and calcified and abscessed hepatic granulomas, with severe infestation by Ascaris lumbricoides and Trichuris trichiura. She was treated with antibiotics and antiparasitic agents with successful recovery.

In our environment, helminth infections are a frequent cause of anemia, stunting and neurodevelopmental delay in school-age children. However, infestation and hepatobiliary complications such as cholangitis, cholecystitis, pancreatitis, biliary lithiasis and hepatic abscess are unusual in children under two years old. Through this case, it is intended to highlight the atypical presentation of this disease at the age of this patient and encourage the strengthening of public health interventions. MÉD.UIS.2020;33(1):67-72.

Keywords: Ascaris lumbricoides. Biliary Tract Diseases. Malnutrition.

Artículo recibido el 28 de abril de 2019 y aceptado para publicación el 19 de marzo de 2020 
¿Cómo citar este artículo?: Bello-Suárez A, González-Valencia G, Sosa L, Sarmiento-Wilches P. Ascariasis hepatobiliar en una lactante con desnutrición severa: reporte de caso. MÉD. UIS.2020;33(1):67-72. doi: 10.18273/revmed.v33n1-2020008

\section{Introducción}

La ascariasis es la geohelmintiasis más prevalente del ser humano. A nivel mundial, afecta a más de 1000 millones de personas y es responsable de alrededor de 20000 muertes anuales ${ }^{1,2}$. Es más frecuente en países en vía de desarrollo que cursan con malas condiciones de saneamiento y pobreza, siendo catalogada como una enfermedad tropical desatendida ${ }^{3-5}$. La prevalencia de helmintiasis en Latinoamérica entre 2000 y 2010 fue de $31,3 \%$ para niños de 1 a 14 años de edad siendo mayor en Brasil, Honduras, Argentina y México, con una mayor frecuencia en preescolares $\left(11,9 \%\right.$ y escolares $(56,7 \%){ }^{6}$ En Colombia, se estima una incidencia de ascariasis de 32,6 casos por 100000 habitantes $^{7}$.

El A. lumbricoides es un nemátodo que reside en el yeyuno donde se reproduce de manera sexuada ${ }^{1-4}$. La hembra genera hasta 200000 huevos diarios ${ }^{8,9}$. La fase terrestre dura entre 10 días y 6 semanas hasta ser infeccioso. A través de alimentos contaminados se ingieren los huevos iniciando así la fase intestinal y tisular de su ciclo vital (hígado, corazón y pulmón), que dura dos meses aproximadamente hasta alcanzar su forma adulta fértil. La infección generalmente es asintomática $\mathrm{o}$ produce manifestaciones leves. El espectro clínico de la enfermedad en niños es inespecífico e incluye: dolor abdominal, naúsea, vómito, inapetencia, fiebre, diarrea, tos, desnutrición, retraso en el crecimiento y desarrollo y anemia ferropénica ${ }^{10-13}$. Complicaciones hepatobiliares, síndrome de Löffler, obstrucción intestinal, peritonitis, pancreatitis y colangitis son secundarias a infestaciones masivas e invasión de órganos, conductos y cavidades (ascariasis errática), las cuales se han descrito también en este grupo de edad ${ }^{1,11,13,14}$.

Si bien, las complicaciones obstructivas digestivas son comunes en niños especialmente entre 4-8 años de edad, las complicaciones hepatobiliares son más frecuentes en adultos, especialmente en mujeres'. Es por ello que el propósito de presentar este caso es el resaltar la presentación atípica de la enfermedad en lactantes y recordar la importancia de fortalecer las intervenciones en salud publica en la región para controlar la morbilidad, reducir la prevalencia de la infección y los costos de intervención de los pacientes con complicaciones ${ }^{15}$. Para la presentación de este caso, se obtuvo el consentimiento informado respectivo por parte del cuidador.

\section{Presentación de caso}

Lactante femenina de 16 meses procedente de zona de extrema pobreza. Consultó por una semana de fiebre, vómito, ausencia de deposiciones, distensión y dolor abdominal. Desde hace un año con salida en múltiples ocasiones de vermes por ano y boca, tratada en numerosas oportunidades con albendazol. Se encontraron como hallazgos positivos al examen físico hipoactividad, taquicardia, fiebre, ojos hundidos, mucosas secas, tiraje subcostal, abdomen distendido y aparentemente doloroso a la palpación, borde hepático a $3 \mathrm{~cm}$ de la reja costal derecha, máculas y pápulas descamativas eritematosas en piel con estigmas de rascado, hipotrofia en extremidades y signo de bandera en cabello. Se diagnosticó pseudo-obstrucción intestinal, desnutrición severa tipo marasmo-kwashorkor, choque mixto por sepsis de origen intra-abdominal, deshidratación grave, hepatomegalia, impétigo no bulloso, escabiosis, pediculosis, retraso del neurodesarrollo, deprivación psicoafectiva y sospecha de abandono. Los laboratorios evidenciaron leucocitosis, proteína C reactiva elevada, anemia moderada, hiponatremia, gamaglutamiltransferasa y fosfatasa alcalina elevadas, hipoalbuminemia moderada e hipoglucemia. Transaminasas, bilirrubinas, tiempos de coagulación, uroanálisis, coproparasitoscópico y radiografía de tórax fueron normales. La radiografía abdominal sugirió pseudoobstrucción intestinal, mientras la ecografía abdominal evidenció infestación de la vía biliar con múltiples imágenes de apariencia tubular en la región del hilio hepático y división derecha de la vía biliar, neumobilia, adenomegalia hiliar hepática, engrosamiento discreto de la pared vesicular, con pequeños focos ecogénicos con densidad de tejido blando en su interior y nodulaciones sugestivas de granulomas calcificados por ascariasis en segmentos VII, V y IVB del hígado, algunas con imágenes tubulares en su interior (Ver Figura 1). 
Enero - abril

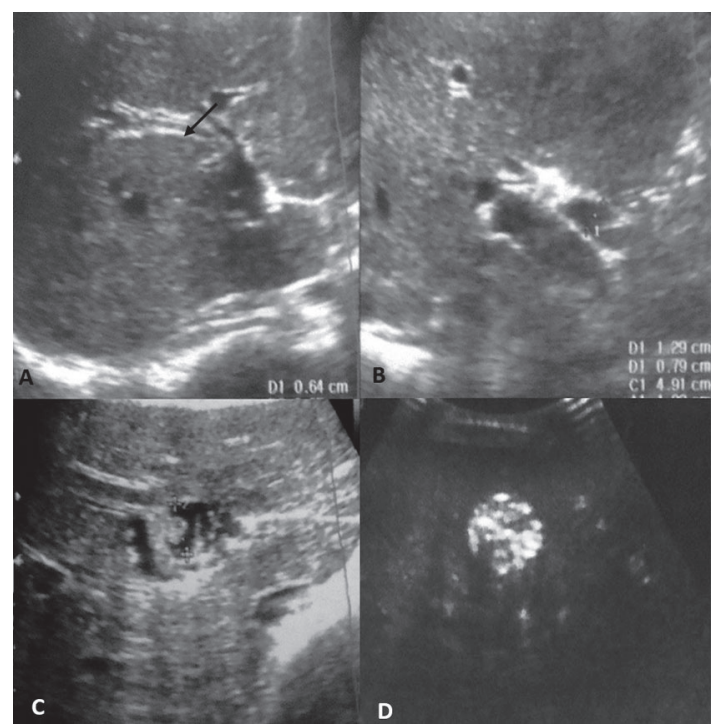

Figura 1. Ecografía hepatobiliar. (A) y (B) Dilatación de vía biliar intrahepática, Flecha señala pared hiperecogénica del parásito, contrastando con la cavidad corporal anecóica del mismo. (C) En corte axial oblicuo se ve una ecogenicidad de tejido blando dentro del conducto biliar dilatado. Probablemente correspondiente a helminto en conducto biliar. (D) Segmento hepático $\mathrm{V}$ con nódulos hiperecogénicos con calcificaciones mixtas de aproximadamente 15 $-18 \mathrm{~mm}$.

Fuente: autores.

Se inició reanimación hídrica, junto a Ampicilina, Amikacina, Metronidazol y Piperazina previos policultivos (hemocultivos, coprocultivo, urocultivo), sonda nasogástrica descompresiva, nutrición parenteral y desimpactación con polietilenglicol. Tras tres días de manejo, hubo salida de ovillo de A. Lumbricoides por heces (Ver Figura 2A y B). Se enviaron 3 muestras seriadas de materia fecal al laboratorio de parasitología de la Universidad Industrial de Santander para conteo de huevos y larvas. Mediante método de Kato-Katz, se informó al segundo día la presencia inicial de 1'372 000 huevos por gramo de materia fecal (hpg-mf) de A. lumbricoides fértiles en su totalidad y $512000 \mathrm{hpg}$ mf de T. trichiura (Ver Figura 2C, D y E), por lo que se adicionó manejo con Pamoato de Pirantel-Oxantel por 3 días. Se suspendieron los antibióticos al quinto día por reporte negativo de policultivos. Prueba VIH ELISA fue negativa, conteo de inmunoglobulinas ( $G$, $M, A$ ), complemento $C_{3}, C_{4}$ y relación de población de linfocitos (CD3-CD4-CD8) fueron adecuados para la edad, mientras la IgE resultó elevada (440 $\mathrm{UI} / \mathrm{mL}$ ). A los 7 días de hospitalización, presentó síndrome febril, eosinofilia (absolutos en 1 599) y aparición de síntomas respiratorios bronco-
Ascariasis hepatobiliar en una lactante con desnutrición severa: reporte de caso

obstructivos autolimitados con nuevos infiltrados parahiliares en la radiografía torácica, hallazgos suficientes para diagnosticar Síndrome de Löffler. Además, por persistencia de hepatomegalia se tomó ecografía de control que reportó ascariasis hepática sobreinfectada con abscesos hepáticos piógenos, se decidió completar tratamiento con Albendazol por 7 días y Ampicilina Sulbactam - Clindamicina por 10 días. Por mejoría clínica, paraclínica e imagenológica, egresó tras 37 días de estancia intrahospitalaria. Debido a que se encontró ascariasis en el núcleo familiar, se indicó tratamiento antihelmíntico y quimioprofilaxis semestral. No se logró seguimiento posterior de la paciente por pérdida de contacto de la madre sustituta con la entidad de salud.

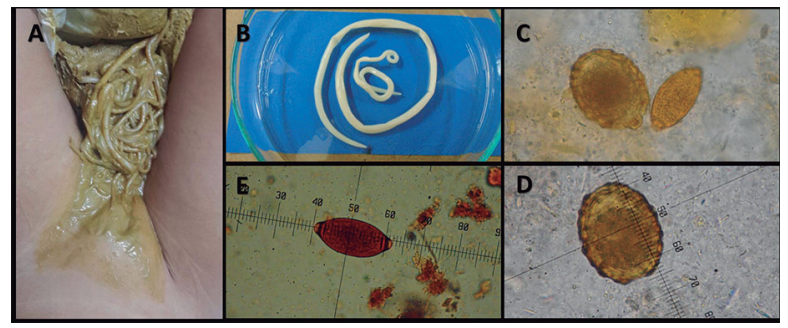

Figura 2. Ascariasis de la paciente. (A) Salida de nudos de Ascaris lumbricoides durante la desimpactación. (B) Macho (Interno) y Hembra (Externa) de Ascaris lumbricoides Jóvenes. (C) 40X Huevo de Ascaris lumbricoides y Huevo de Trichuris trichiura. (D) $40 \mathrm{X}$ Huevo de Ascaris lumbricoides (65 um) (E) 40X Huevo de Trichuris trichiura (55 um)

Fuente: autores.

\section{Discusión}

La ascariasis es una patología prevalente en pediatría, destacándose 2 aspectos en este grupo etáreo: el grado de infestación usualmente es leve a moderado y la obstrucción intestinal como complicación gastrointestinal es más frecuente; mientras que en edades adultas son más comunes las infestaciones severas y las complicaciones hepatobiliares ${ }^{1,11}$.

La migración errática biliar del helminto, es una complicación frecuente en adultos (53\%) pero infrecuente en niños (5\%). Puede generar obstrucción mecánica, espasmo o dismotilidad del esfínter de Oddi transitoria o permanente, con una intensa respuesta inflamatoria y necrótica localizada llevando a colangitis, colecistitis, pancreatitis, litiasis biliar, peritonitis biliar por perforación del conducto hepático común o hepatitis abscedada ${ }^{1,11-14}$. Las reinfecciones hepáticas recurrentes pueden derivar en atrofia, cirrosis y falla hepática,11,12. Las principales manifestaciones en niños son: dolor 
abdominal (100\%), vómito (96\%), visualización del gusano en heces o por expulsión oral (96\%), fiebre (27\%), hepatomegalia (16\%) e ictericia (2\%) $)^{11,13}$. La mayoría de ellas reportadas en esta paciente.

El diagnóstico se realiza mediante la identificación de huevos en examen directo, método Beaver, o mediante técnicas de concentración, Ritchie o KatoKatz. Esta última, recomendada por la Organización Mundial de la Salud (OMS) por su mayor sensibilidad. Según el conteo de huevos se categoriza la infección en leve ( $<5000 \mathrm{hpg}-\mathrm{mf}$ ), moderada (entre $5000 \mathrm{y}$ 50000 hpg-mf) y severa (>50 000 hpg-mf) $)^{5,16,17}$. En nuestro caso se documentó un conteo de más de un millón de hpg-mf, significando una infestación masiva. La detección de antígenos por reacción en cadena de polimerasa, hemoaglutinación indirecta y aglutinación en látex permiten su genotipificación, pero por su alto costo se reservan para investigaciones ${ }^{3}$.

En el estudio imagenológico para las complicaciones hepatobiliares en niños se prefiere la ecografía por su accesibilidad y buen rendimiento; los hallazgos descritos son: la identificación de una estructura tubular anecoica con paredes hiperecogénicas en vía biliar (señal de tubo interno), distensión de la vesícula biliar con estructura tubular enrollada, dilatación de los conductos con el signo de la tira o del espagueti, abscesos hepáticos y calcificaciones ${ }^{1,18,19}$. La Colangiopancreatografía Retrógrada Endoscópica (CPRE) se reserva para casos con alta sospecha de parasitosis en vía biliar y ecografía normal, siendo el gold estándar diagnóstico'. Otros hallazgos son la eosinofilia y los cristales de Charcot-Leyden en muestras de heces o esputo ${ }^{2,11,13}$.

Las opciones terapéuticas incluyen el tratamiento conservador, la extracción por CPRE y cirugía ${ }^{12,18}$. El tratamiento conservador contempla el uso de antibióticoterapia, antihelmínticos y antiespasmódicos ${ }^{18}$. Cuando la infestación es leve, se puede usar Albendazol, Mebendazol o Pamoato de Pirantel-Oxantel; la Piperazina es la opción de manejo en caso de obstrucción intestinal, ya que induce parálisis flácida del parásito facilitando su expulsión por el peristaltismo intestinal, con un éxito en alrededor del $80 \%$ de los $\operatorname{casos}^{9,13,14,20}$. La mayoría de los pacientes responden con un tiempo de tratamiento de 3 a 5 días, aunque se ha descrito la necesidad de extenderlo hasta 7 días adicionales, en casos de infestaciones severas de difícil manejo ${ }^{18}$.
La intervención quirúrgica se reserva para casos de ascariasis hepatobiliar masiva, abscesos piógenos secundarios, colecistitis y colangitis que no responden a tratamiento conservador ${ }^{18,21}$. La CPRE es una excelente alternativa previa a la cirugía, cuya utilidad es diagnóstica y terapéutica al eliminar los vermes y descomprimir el árbol biliar. No obstante, su uso en pediatría es reservado al ser un procedimiento invasivo de disponibilidad restringida, con limitado personal capacitado para su realización en países de desarrollo intermedio y consiguiente morbilidad elevada asociada'.

El manejo del caso fue conservador, requiriendo una primera fase con Piperazina por 10 días a fin de evitar su migración errática, complementado con Pamoato de Pirantel-Oxantel. El uso de Albendazol, aprobado por la OMS para mayores de 12 meses, al ser eficiente larvicida, ovicida y vermicida en infecciones tisulares sistémicas, se extendió hasta 7 días de acuerdo a la respuesta clínica y parasitoscópica. No se evidenciaron reacciones adversas.

Por otro lado, el estado nutricional deficitario genera susceptibilidad a infección y reinfección por geohelmintos; mientras, la respuesta inmunológica crónica en las reinfestaciones, lleva a un estado inflamatorio regulado a la baja, mediado por IL-10, que disminuye la capacidad de eliminación parasitaria ${ }^{2,4}$. También se ha demostrado que la ascariasis tiene implicaciones en el crecimiento y desarrollo con bajos resultados cognitivos, perpetuando el ciclo de desnutrición, enfermedad y pobreza ${ }^{8,22-24}$.

En el caso de la paciente, llama la atención el grado de infestación presentado, ya que revela un contacto muy temprano y recurrente con el parásito (desde los primeros tres meses de vida aproximadamente), tiempo inferido por la cronología de los síntomas y la duración del ciclo del nematodo; situación que condujo, además del compromiso nutricional severo, a complicaciones hepatobiliares inusuales en esta edad. Cabe resaltar que su infestación severa está vinculada con una alta tasa de producción de huevos fértiles, como se comprobó en las muestras evaluadas. Las condiciones precarias no controladas en las que habitaba la menor y la privación de la lactancia materna contribuyeron a que el ciclo del parásito se perpetuara. De allí que la ascariasis sea un indicador de pobreza y problemas importantes en el saneamiento básico, la higiene y la educación. 
Enero - abril

Las intervenciones preventivas incluyen: lavado de manos, uso de calzado, filtrado o hervor del agua para su consumo y la disposición de excretas. La disposición de acueducto se ha asociado a menor probabilidad de infecciones parasitarias ${ }^{2,25}$. Asimismo, las estrategias de educación para lograr cambios de hábitos y estilos de vida saludables, principalmente la lactancia materna en los primeros 6 meses de vida y el lavado de manos y alimentos, disminuyen en conjunto la posibilidad de infección parasitaria $^{26}$.

El objetivo propuesto por la OMS es reducir la prevalencia de geohelmintiasis de más de 50\% a menos del $20 \%$ en escolares de áreas endémicas a través de modelos establecidos por la resolución CD49.R19 del 2009 de la OMS/OPS 25,27. Las estrategias preventivas actuales se centran en administrar quimioprofilaxis con antihelmínticos (Albendazol - Mebendazol), pues no solo reducen la carga parasitaria y alivian los síntomas, sino que también reducen la tasa de transmisión. En zonas de alta endemicidad se recomienda la desparasitación una vez al año si la prevalencia supera el 20\% y dos veces al año si supera el $50 \% 25$. Sin embargo, esta intervención es útil y efectiva a corto plazo, ya que con el tiempo las condiciones medioambientales desfavorables permiten la reinfección ${ }^{27}$. Aunque es tema ampliamente conocido, es importante enfatizar en las políticas y estrategias de salud pública básicas para mantener las condiciones mínimas de salud de la población.

\section{Conclusión}

La ascariasis es una enfermedad desatendida que genera coomorbilidades graves en la población infantil como este caso inusual de infestación, desnutrición y complicaciones hepatobiliares en una menor de dos años vinculado a un ambiente de abandono e insalubridad. Algunas de las complicaciones presentadas por esta paciente son consideradas eventos de importancia en salud pública que al ser reportados a las entidades de vigilancia y registro gubernamental, derivan en información que determina la movilización de recursos para garantizar condiciones básicas de salud y favorecer el crecimiento y desarrollo de la infancia en la región y el país; lo cual se traduce en mejoría en los indicadores de desarrollo.
Ascariasis hepatobiliar en una lactante con desnutrición severa:

reporte de caso

\section{Declaración de conflictos de interés}

No tenemos conflictos de interés y para la financiación de la elaboración de este artículo declaramos que no utilizamos ayudas económicas externas ni financiación alguna.

\section{Agradecimientos}

Francisco Tirado Santamaría

Profesor Catedrático de la Universidad Industrial de Santander

BLC, Esp en Epidemiología, Msc DDHH.

Luis Eduardo Franco Ospina

Profesor Catedrático de la Universidad Industrial de Santander

Médico, Radiólogo.

\section{Referencias bibliográficas}

1. Khuroo MS, Rather AA, Khuroo NS, Khuroo MS. Hepatobiliary and pancreatic ascariasis. World J Gastroenterol. 2016;22(33):75077517. doi:10.3748/wjg.v22.i33.7507

2. Cooper PJ. Mucosal immunology of geohelminth infections in humans. Mucosal Immunol. 2009;2(4):288-299. doi:10.1038/ mi.2009.14

3. Knopp S, Steinmann P, Keiser J, Utzinger J. Nematode Infections. Infect Dis Clin N Am. 2012;26(2):341-358. doi:10.1016/j. idc.2012.02.006

4. Cooper PJ, Figuieredo CA. Immunology of Ascaris and Immunomodulation.; 2013. doi:10.1016/B978-0-12-3969781.00001-X

5. Smith HM, DeKaminsky RG, Niwas S, Soto RJ, Jolly PE. Prevalence and Intensity of Infections of Ascaris lumbricoides and Trichuris trichiura and Associated Socio-demographic Variables in Four Rural Honduran Communities. Mem Inst Oswaldo Cruz. 2001;96(3):303-314. doi:10.1590/S0074-02762001000300004

6. Saboyá M, Catalá L, Nicholls R, Ault S. Update on the Mapping of Prevalence and Intensity of Infection for Soil-Transmitted Helminth Infections in Latin America and the Caribbean : A Call for Action. PLoS Negl Trop Dis. 2015;7(9):1-10. doi:10.1371/ journal.pntd.0002419

7. Rodriguez-Morales AJ, Valencia-Salinas M, Murillo-Abadia J, et al. Ascariasis among Internally Displaced People of Colombia, 2009-2016. Int J ofInfectious Dis 73S. 2018;3:227. doi:10.1016/j. ijid.2018.04.3930

8. Hlaing T. Ascariasis and childhood malnutrition. Parasitology. 1993;(107):S125-S136. doi:10.1016/0035-9203(92)90133-W

9. Dent AE, J. K. Ascariasis ( Ascaris Lumbricoides ). 20th ed. (Kliegman R, Stanton B, St. Geme J, Schor N, eds.). Barcelona: Elsevier; 2016.

10. Shoff W. Pediatric Ascariasis: Background, Pathophysiology, Epidemiology. Medscape.

11. Soares M da G, Baldisseroto M, Custodio CM, Gralha CZ, Mangili AR. Hepatobiliary and Pancreatic Complications of Ascariasis in Children : A Study of Seven Cases. J Pediatr Gastroenterol Nutr. 2001;33(September):271-275.

12. Eyer de Jesus L, Pecoraro R R, Guazelli A. Biliary Ascariasis Spectrum of Surgical Problems and Tactics. Rev Col Bras Cir. 2004;31(3):172-179 
13. Grijalva DS. Manifestaciones Clínicas, Diagnóstico y Tratamiento del Áscaris lumbricoides en Pediatría. Rev Gastrohnup. 2016;18:s1, e23-e31.

14. Dall' Orso P, Cantou V, Rosano K, et al. Ascaris lumbricoides, Complicaciones graves en niños hospitalizados en el Centro Hospitalario Pereira Rossell. Arch Pediatr Urug. 2014;85(3):149-154.

15. Jourdan PM, Lamberton PHL, Fenwick A, Addiss DG. Soiltransmitted helminth infections. Lancet. 2018;391(10117):252 265. doi:10.1016/S0140-6736(17)31930-X

16. Restrepo Von Schiller IC, Mazo Berrío LP, Salazar Giraldo ML, Montoya Palacio MN, Botero Garcés JH. Evaluación de tres técnicas coproparasitoscópicas para el diagnóstico de geohelmintos intestinales. Iatreia. 2013;26(1):15-24.

17. Periago M V, Diniz RC, Pinto SA, et al. The Right Tool for the Job: Detection of Soil- Transmitted Helminths in Areas Coendemic for Other Helminths. PLoS Negl Trop Dis. 2015;9(8):115. doi:10.1371/journal.pntd.0003967

18. Lynser D, Marbaniang E. Hepatobiliary and pancreatic ascariasis - sonographic pictorial essay on four pediatric cases with acute biliary colic. Emerg Radiol. 2016;23(2):197-200. doi:10.1007/ s10140-015-1369-7

19. Koumanidou C, Manoli E, Anagnostara A, Polyviou P, Vakaki M. Sonographic features of intestinal and biliary ascariasis in childhood : case report and review of the literature. Ann Trop Paediatr. 2004;24:329-335. doi:10.1179/027249304225019154

20. Ospina J, Posada ME. Manejo endoscópico de la ascaridiasis múltiple biliar en niños . Presentación de casos y revisión de la literatura. Rev Col Gastroenterol. 2007;22(2):149-154.
21. Beckingham IJ, Cullis SN, Krige JEJ, Bornman PC, Terblanche J. Management of hepatobiliary and pancreatic Ascaris infestation in adults after failed medical treatment. Br J Surg. 1998;85(December 1997):907-910.

22. Yap P, Utzinger J, Hattendorf J, Steinmann P. Influence of nutrition on infection and re-infection with soil-transmitted helminths : a systematic review. Parasit Vectors. 2014;7(1):1-14. doi:10.1186/1756-3305-7-229

23. Jardim-Botelho A, Raff S, Ávila Roddrigues R, et al. Hookworm , Ascaris lumbricoides infection and polyparasitism associated with poor cognitive performance in Brazilian schoolchildren. Trop Med Int Heal. 2008;13(8):994-1004. doi:10.1111/j.13653156.2008.02103.x

24. Labeaud AD, Singer MN, Mckibben M, et al. Parasitism in Children Aged Three Years and Under : Relationship between Infection and Growth in Rural Coastal Kenya. PLoS Negl Trop Dis. 2015;21:1-15. doi:10.1371/journal.pntd.0003721

25. WHO. Soil-transmitted helminth infections. WHO. https://www. who.int/news-room/fact-sheets/detail/soil-transmitted-helminthinfections. Published 2019. Accessed December 5, 2019.

26. Strunz EC, Addiss DG, Stocks ME, Ogden S, Utzinger J, Freeman MC. Water, Sanitation, Hygiene, and Soil-Transmitted Helminth Infection: A Systematic Review and Meta-Analysis Eric. PLoS Med. 2014;11(3):1-38. doi:10.1371/journal.pmed.1001620

27. WHO, PAHO. ELIMINACIÓN DE LAS ENFERMEDADES DESATENDIDAS Y OTRAS INFECCIONES RELACIONADAS CON LA POBREZA. PAHO; 2009:1-7. https://www.paho.org/hq/ dmdocuments/2009/CD49.R19 (Esp.).pdf. 